Journal of Nonlinear Mathematical Physics

\title{
Complex Representation of Planar Motions and Conserved \\ Quantities of the Kepler and Hooke Problems
}

Y. Grandati, A. Bérard, H. Mohrbach

To cite this article: Y. Grandati, A. Bérard, H. Mohrbach (2010) Complex Representation of Planar Motions and Conserved Quantities of the Kepler and Hooke Problems, Journal of Nonlinear Mathematical Physics 17:2, 213-225, DOI:

https://doi.org/10.1142/S1402925110000726

To link to this article: https://doi.org/10.1142/S1402925110000726

Published online: 04 January 2021 


\title{
COMPLEX REPRESENTATION OF PLANAR MOTIONS AND CONSERVED QUANTITIES OF THE KEPLER AND HOOKE PROBLEMS
}

\author{
Y. GRANDATI* A. BÉRARD ${ }^{\dagger}$ and H. MOHRBACH ${ }^{\ddagger}$ \\ Institut de Physique, ICPMB, IF CNRS 2843, Université Paul Verlaine \\ 1 Bvd Arago, 57078 Metz, Cedex 3, France \\ *grandati@univ-metz.fr \\ †aberard@univ-metz.fr \\ ${ }^{\ddagger}$ mohrbach@univ-metz.fr
}

Received 29 September 2009

Accepted 15 November 2009

\begin{abstract}
Using a complex representation of planar motions, we show that the dynamical conserved quantities associated to the isotropic harmonic oscillator (Fradkin-Jauch-Hill tensor) and to the Kepler's problem (Laplace-Runge-Lenz vector) find a very simple and natural interpretation. In this frame we also establish in an elementary way the relation which connects them.
\end{abstract}

Keywords: Planar motions; Bohlin-Arnold-Vassiliev duality; complex representation; conserved quantities.

\section{Introduction}

In 1873 J. Bertrand [3, 10] established that, among all central potentials, the Kepler $(1 / r)$ and Hooke $\left(r^{2}\right)$ sytems are very specific in the sense that they are the only ones for which, at the classical level, the bounded planar orbits are closed for any initial conditions and of elliptic type. This specificity is met again in the fact that, besides the energy and angular momentum, they possess additional globally conserved quantities, respectively of vectorial (Laplace-Runge-Lenz vector) and tensorial (Fradkin-Jauch-Hill tensor) natures [7, 19, 21]. These supplementary constants of motion are related to the existence of a dynamical group of symmetries larger than the space-time one for both systems.

The study of a relation connecting these two privileged systems has a long history initiated in Hooke and Newton's works [13]. In 1911 Bohlin showed that this relation can be formulated in terms of conformal mapping [4]. This conformal transform is at the core of the Levi-Civita's regularization scheme [22] of which tridimensional generalization has been achieved by Kustaanheimo and Stiefel [34]. In fact, as noted by Needham [26, 27] two years before Bohlin's paper, Kasner [20] had established a more general duality law relating pairs of power law potentials (but the result has only been published in 1913). This relation has been rediscovered and generalized by Arnold and Vassiliev [1, 2] in 1989 and 
quite simultaneously by Hojman et al. [17]. In fact the generalization of Kasner's result had already been obtained by Collas [5] and implicitly enters into the frame of the coupling constant metamorphosis of Hietarinta et al. [16, 31, 37]. Even if we restrict ourselves to the classical aspects (the study of this correspondence in the quantum mechanical frame has an interesting parallel history that we do not deal with here), numerous articles have been published on this subject during the last fifteen years $[13,15,24,25,35,36,38]$.

In this article we show that the use of a complex representation, particularly well adapted in the study of planar motions, permits one to give a very simple and transparent interpretation to the Jauch-Hill-Fradkin (JHF) tensor and Laplace-Runge-Lenz (LRL) vector. In the first part, we begin by giving the essentials of the complex representation scheme. Treating Hooke and Kepler systems in this frame, we show that the FJH tensor and the LRL vector are induced by the existence of simple integrating factors for the respective equations of motion. In particular, for the Hooke system, it is directly induced by the analytic form of the equation of motion, which is peculiar to this system.

We then recall the principle of Bohlin-Arnold-Vassiliev's dual correspondence. Applying it to the special case of the Hooke-Kepler connection, we then recover in a simple and direct way the link established by Nersessian et al. [28-30] between the additional dynamical conserved quantities associated to these systems. Moreover, it appears clearly that the generators of the dynamical symmetry algebras of the Kepler and Hooke systems respectively, are (up to a constant factor) the same object, expressed in the systems of representation (coordinates and parametrization) associated to the two dual motions.

\section{Complex Formulation of Planar Motions}

\subsection{Basic elements}

We are interested in the study of the motion $\vec{r}(t)$ for a particle of mass $m$ subjected to a central potential $U(\vec{r})=U(r)$, eventually singular at the origin for which the equation of motion is given by:

$$
m \ddot{\vec{r}}=-\vec{\nabla} U(r)=-U^{(1)}(r) \frac{\vec{r}}{r}
$$

where the dot represents the time $t$ derivative and $U^{(n)}(r)=d^{n} U(r) / d r^{n}$.

As it is well known, the angular momentum $\vec{L}=m \vec{r} \times \dot{\vec{r}}$ of the system is conserved:

$$
\dot{\vec{L}}=m \vec{r} \times \ddot{\vec{r}}=\overrightarrow{0}
$$

and the motion is contained in the plane $\left(O, \vec{u}_{1}, \vec{u}_{2}\right)$ orthogonal to $\vec{L}=L \vec{u}_{3},\left(O, \vec{u}_{1}, \vec{u}_{2}, \vec{u}_{3}\right)$ being an orthogonal frame of the three-dimensional space.

To describe such a planar motion, we can adopt a complex formulation and represent any vector $\vec{A}$ in the plane of the motion by a corresponding complex number $A$, called its affix:

$$
\vec{A}=\left(\begin{array}{c}
A_{1} \\
A_{2}
\end{array}\right)_{\left(O, \vec{u}_{1}, \vec{u}_{2}\right)} \rightarrow A=A_{1}+i A_{2} \in \mathbb{C}
$$


For the position vector, we have in particular:

$$
\vec{r}(t)=\left(\begin{array}{l}
x(t) \\
y(t)
\end{array}\right)_{\left(O, \vec{u}_{1}, \vec{u}_{2}\right)} \rightarrow z(t)=x(t)+i y(t) .
$$

In the plane of motion the potential is viewed as a real-valued function, $U(z, \bar{z})$ of $z$ and $\bar{z}$, the gradient of which is given by:

$$
\vec{\nabla} U(\vec{r}) \rightarrow 2 \frac{\partial U(z, \bar{z})}{\partial \bar{z}},
$$

where:

$$
\left\{\begin{array}{l}
\frac{\partial}{\partial \bar{z}}=\frac{1}{2}\left(\frac{\partial}{\partial x}-i \frac{\partial}{\partial y}\right) \\
\frac{\partial}{\partial \bar{z}}=\frac{\partial}{\partial z} .
\end{array}\right.
$$

In this representation, the equation of motion takes the form:

$$
\ddot{z}+\frac{2}{m} \frac{\partial U(z, \bar{z})}{\partial \bar{z}}=0 .
$$

For a central potential, it becomes simply:

$$
\ddot{z}+\frac{1}{m} U^{(1)}(r) \frac{z}{r}=0 .
$$

Multiply both sides of Eq. (7) by $\dot{\bar{z}}$ and both sides of the complex conjugate equation by $\dot{z}$. Then adding the resulting identities, we obtain:

$$
\frac{\dot{\bar{z}} \ddot{z}+\dot{z} \ddot{\bar{z}}}{2}+\frac{1}{m}\left(\dot{\bar{z}} \frac{\partial U(z, \bar{z})}{\partial \bar{z}}+\dot{z} \frac{\partial U(z, \bar{z})}{\partial z}\right)=0
$$

the left member of which is a total derivative.

Integrating this identity with respect to $t$, we recover the conservation of energy:

$$
E=\frac{1}{2} m|\dot{z}|^{2}+U(z, \bar{z})=\frac{1}{2} m\|\dot{\vec{r}}\|^{2}+U(\vec{r}) .
$$

If $\vec{A}$ and $\vec{B}$ are two vectors in the $\left(O, \vec{u}_{1}, \vec{u}_{2}\right)$ plane, the complex affix of $\vec{A} \times \vec{B}$ is given by the real quantity $\operatorname{Im}(\bar{A} B), A$ and $B$ being the complex affixes of $\vec{A}$ and $\vec{B}$, respectively (this real quantity corresponds to a vector which is orthogonal to the $\left(O, \vec{u}_{1}, \vec{u}_{2}\right)$ plane).

The angular momentum $\vec{L}$ admits the real affix:

$$
L=m \operatorname{Im}(\bar{z} \dot{z})=\frac{m}{2 i}(\bar{z} \dot{z}-\dot{\bar{z}} z) \in \mathbb{R} .
$$

Note that for any vector $\vec{A}$ lying in the $\left(O, \vec{u}_{1}, \vec{u}_{2}\right)$ plane we have the following correspondence:

$$
\vec{L} \times \vec{A}=-L A_{2} \vec{u}_{1}+L A_{1} \vec{u}_{2} \rightarrow i L A
$$




\subsection{Isotropic harmonic oscillator (Hooke's problem)}

We consider the case for which the second term, $\partial U(z, \bar{z}) / \partial \bar{z}$, of the equation (7) is analytic in $z$, that is the equation not $\bar{z}$ dependent:

$$
\ddot{z}+\frac{1}{m} a(z)=0 .
$$

Equation (7) admits an integrating factor $\dot{z}$ and besides $E$ and $L$ we obtain after a staightforward integration the following complex conserved quantity:

$$
\mathcal{F}=m \frac{(\dot{z})^{2}}{2}+\int a(z) d z
$$

If we restrict ourselves to central potentials, we see immediately that this analycity condition corresponds to $U^{(1)}(r) / r$ (see Eq. (8)) constant in $r$, that is to the Hooke potential (isotropic harmonic oscillator) $U(z, \bar{z})=k z \bar{z} / 2, k \in \mathbb{R}$.

We then have $a(z)=k z$ and Eq. (8) is linear:

$$
\ddot{z}+\frac{k}{m} z=0
$$

(and its solution is straightforwardly obtained as a linear combination of the two basis solutions $\exp ( \pm i \sqrt{k / m} t))$.

$F$ becomes simply (see Eq. (14)):

$$
\mathcal{F}=m \frac{(\dot{z})^{2}}{2}+\frac{k}{2} z^{2}
$$

or in algebraic form:

$$
\mathcal{F}=\mathcal{F}_{1}+i \mathcal{F}_{2}
$$

where:

$$
\mathcal{F}_{1}=T_{x x}-T_{y y}, \quad \mathcal{F}_{2}=T_{x y}
$$

$T=\left(T_{i j}\right)_{i, j \in\{x, y\}}$ being the well known Jauch-Hill-Fradkin tensor [7, 19]:

$$
T=\left(\begin{array}{cc}
T_{x x} & T_{x y} \\
T_{x y} & T_{y y}
\end{array}\right)=\left(\begin{array}{cc}
m(\dot{x})^{2} / 2+k x^{2} / 2 & m \dot{x} \dot{y} / 2+k x y / 2 \\
m \dot{x} \dot{y} / 2+k x y / 2 & m(\dot{y})^{2} / 2+k y^{2} / 2
\end{array}\right) .
$$

The energy is the trace of this tensor: $E=\operatorname{Tr}(T)=m|\dot{z}|^{2} / 2+k|z|^{2} / 2$ and we can write:

$$
\left\{\begin{array}{l}
T_{x x}=\frac{1}{2}\left(E+\mathcal{F}_{1}\right) \\
T_{y y}=\frac{1}{2}\left(E-\mathcal{F}_{1}\right) \\
T_{x y}=\mathcal{F}_{2} .
\end{array}\right.
$$


Note that, if we define

$$
F_{1}=\frac{1}{\omega} \mathcal{F}_{2}, \quad F_{2}=\frac{1}{2 \omega} \mathcal{F}_{1}, \quad F_{3}=\frac{1}{2} L
$$

with $\omega=\sqrt{k / m}$, it can be easily shown that the $F_{i}$ satisfy the following Poisson Brackets relations $[14,19,23]$ :

$$
\left\{F_{i}, F_{j}\right\}=\varepsilon_{i j k} F_{k}
$$

and then are generators of the dynamical $\mathrm{O}(3)$ symmetry algebra associated to the isotropic harmonic oscillator.

The complex representation allows one to show the existence of a specific conserved quantity for the harmonic oscillator and of the associated global dynamical symmetry in a straightforward way. The use of complex numbers rather than vectors permits one to profit from their algebraic properties. The existence of the Jauch-Hill-Fradkin tensor and of the associated dynamical symmetry appears in a transparent way, as a very simple consequence of the analytic in $z$ dependence of the equation of motion which is a characteristic feature of the Hooke system.

\subsection{Kepler's problem}

Besides the isotropic harmonic oscillator there is only one other one-particle central system which admits a globally defined conserved quantity, namely the Kepler system $U_{K}(r)=$ $-\widetilde{k} / r$. For it, the conserved quantity is the well known Laplace-Runge-Lenz vector [21]. One may wonder if it can be obtained via a simple and direct quadrature on the associated equation of motion:

$$
\ddot{z}+\frac{\widetilde{k}}{m} \frac{z}{r^{3}}=0
$$

as it was the case for the Hooke problem (see Eq. (15)).

Looking for an integrating factor, we can think to use a quantity in which any term contains $\dot{z}$ or $\dot{\bar{z}}$ as a factor. This is the case of $i L=m(\bar{z} \dot{z}-\dot{\bar{z}} z) / 2$ (see Eq. (11)). Multiplying both sides of Eq. (22), we obtain:

$$
i L \ddot{z}+\frac{\widetilde{k}}{2}(\bar{z} \dot{z}-\dot{\bar{z}} z) \frac{z}{r^{3}}=0 .
$$

As $L$ being conserved, the first term is the total time derivative of $(i L \dot{z})$. As for the second term it can be rewritten as:

$$
\frac{\widetilde{k}}{2}(\bar{z} \dot{z}-\dot{\bar{z}} z) \frac{z}{r^{3}}=\frac{\widetilde{k}}{2}\left(\frac{\dot{z}}{z}-\frac{\dot{\bar{z}}}{\bar{z}}\right) \sqrt{\frac{z}{\bar{z}}}=\widetilde{k} \frac{d}{d t}\left(\sqrt{\frac{z}{\bar{z}}}\right)=\widetilde{k} \frac{d}{d t}\left(\frac{z}{r}\right) .
$$

Then the left-hand member of Eq. (23) is a total time derivative and we obtain the following conserved quantity:

$$
\mathcal{A}=i L \dot{z}+\widetilde{k} \frac{z}{r}
$$


Using Eq. (12), we immediately recognize the affix of the well-known Laplace-RungeLenz vector [21] for the Kepler motion $\vec{r}(t)$ :

$$
\overrightarrow{\mathcal{A}}=\vec{L} \times \dot{\vec{r}}+\widetilde{k} \frac{\vec{r}}{r}
$$

Again, due to the algebraic properties of complex entities, the existence of the LaplaceRunge-Lenz vector appears as simply induced by the existence of a simple, although nonanalytical, integrating factor, namely the angular momentum, for the equation of motion. Note that the components of $\overrightarrow{\mathcal{A}}$ are the generators of the dynamical su(2) symmetry algebra associated to the two-dimensional Kepler problem.

In complex representation Hamilton's vector $[8,21,33]$ is simply obtained as:

$$
\mathcal{K}=\frac{i \mathcal{A}}{L}=\frac{\widetilde{k}}{L} \frac{i z}{r}-\dot{z}
$$

or in, using Eqs. (25) and (12):

$$
\overrightarrow{\mathcal{K}}=\frac{1}{L^{2}} \vec{L} \times \overrightarrow{\mathcal{A}}=\frac{\widetilde{k}}{L} \vec{e}_{\theta}-\dot{\vec{r}}
$$

(iz/r being the affix of the $\vec{e}_{\theta}=\vec{u}_{3} \times \vec{r} / r$ vector of polar coordinates). Its constancy is then a direct consequence of these ones of $\vec{L}$ and $\overrightarrow{\mathcal{A}}$.

$\mathcal{K}$ can also be obtained directly using Eqs. (11) and (24). Their combination imply indeed:

$$
\frac{i L}{m} \frac{\widetilde{k} z}{r^{3}}=\widetilde{k} \frac{d}{d t}\left(\frac{z}{r}\right)
$$

which permits to write the left member of Eq. (22) as a total derivative without having to introduce any integrating factor:

$$
\frac{d}{d t}\left(\dot{z}+\frac{\widetilde{k}}{i L} \frac{z}{r}\right)=0 .
$$

We deduce immediately:

$$
i \frac{\widetilde{k}}{L} \frac{z}{r}-\dot{z}=\mathcal{K}=\text { const. }
$$

\section{Complex Transformations and Bohlin-Arnold-Vassiliev's Duality}

In the preceding section we have obtained a unified interpretation of the origin of specific conseved quantities for the Hooke and Kepler systems. In both cases it is simply connected to the existence of simple integrating factors for the complex formulated equation of motion. The question which of course arises is to know if this common behavior is due to an underlying relation between the two systems. The answer has been given one century ago by Levi-Civita [22], Bohlin [4] and Kasner [20] and recently settled in a more general frame by Arnold and Vassiliev [1,2]. This relation appears in fact as a particular case of the so-called Bohlin-Arnold-Vassiliev's duality which relates a class of planar motions to 
which the power law potentials belong. This duality scheme appears very naturally if we consider the possible transformations of the equations of motion combining an analytical change of the complex coordinate and a local reparametrization of the motion.

\subsection{Conformal change of coordinates}

We return to the general equation (7) and consider firstly an arbitrary conformal (that is analytical) change of coordinates:

$$
z=f(w), \quad \bar{z}=\overline{f(w)}=\bar{f}(\bar{w}) .
$$

Then

$$
\frac{\partial}{\partial \bar{z}}=\left(\frac{\partial \bar{z}}{\partial \bar{w}}\right)^{-1} \frac{\partial}{\partial \bar{w}}=\frac{1}{\bar{f}^{(1)}(\bar{w})} \frac{\partial}{\partial \bar{w}}=\frac{1}{\overline{f^{(1)}(w)}} \frac{\partial}{\partial \bar{w}}
$$

where $f^{(n)}(z)=\partial^{n} f(z) / \partial z^{n}$.

After substitution, Eq. (7) takes the following form:

$$
\ddot{w}+(\dot{w})^{2} \log \left(f^{(1)}(w)\right)^{(1)}+\frac{2}{m} \frac{1}{\left|f^{(1)}(w)\right|^{2}} \frac{\partial \widetilde{U}(w, \bar{w})}{\partial \bar{w}}=0
$$

where $\widetilde{U}(w, \bar{w})=U(f(w), \bar{f}(\bar{w}))$.

\subsection{Euler-Sundman's reparametrization}

Equation (34) above incorporates now a term of first order in time derivative, which has an artificial character. In order to suppress it, we can envisage to change the parametrization for the motion $w(t)$. A global change of parametrization is inefficient. Rather, we choose a change of parametrization having a local structure, that is an Euler-Sundman's reparametrization of the type:

$$
d t=|g(w)|^{2} d s
$$

where $g(w)$ is an arbitrary analytic function. This form ensures in particular that the correspondence between the initial time parameter $t$ and the new (fictitious) time $s$ is one-to-one and increasing, with

$$
\frac{d}{d t}=|g(w)|^{-2} \frac{d}{d s}
$$

Starting from Eq. (34), it is straightforward to show that the equation for the motion in $w(s)$ is now given by:

$$
w^{\prime \prime}+\left(w^{\prime}\right)^{2}\left(\log \frac{f^{(1)}(w)}{g(w)}\right)^{(1)}-\overline{(\log g(w))^{(1)}}\left|w^{\prime}\right|^{2}+\frac{2}{m} \frac{|g(w)|^{4}}{\left|f^{(1)}(w)\right|^{2}} \frac{\partial \widetilde{U}(w, \bar{w})}{\partial \bar{w}}=0 .
$$

A priori the structure of this equation is still more complicated than the previous one since it incorporates two kinds of terms depending upon the first derivative in time: the first one depending upon $\left(w^{\prime}\right)^{2}$ as in Eq. (34) and the second one, depending upon $\left|w^{\prime}\right|^{2}$. 
But the gain becomes readily clear. Indeed the freedom in the choice of $g(w)$ allows us to expect to suppress the first one. As for the problem induced by the second, as we just go to see, it is solved by the use of energy conservation identity.

\subsection{Energy conservation}

Expressed in terms of $w$ and $s$, the energy conservation equation (10) gives:

$$
\left|w^{\prime}\right|^{2}=\frac{2}{m}(E-\widetilde{U}(w, \bar{w})) \frac{|g(w)|^{4}}{\left|f^{(1)}(w)\right|^{2}} .
$$

We are then in position to replace in Eq. (37) the contribution containing $\left|w^{\prime}\right|^{2}$ by another one, depending only on the position. We obtain:

$$
\begin{aligned}
w^{\prime \prime}+ & \left(w^{\prime}\right)^{2}\left(\log \left(\frac{f^{(1)}(w)}{g(w)}\right)\right)^{(1)} \\
& +\frac{2}{m} \frac{|g(w)|^{4}}{\left|f^{(1)}(w)\right|^{2}}\left(\frac{\partial \widetilde{U}(w, \bar{w})}{\partial \bar{w}}-\overline{(\log g(w))^{(1)}}(E-\widetilde{U}(w, \bar{w}))\right)=0 .
\end{aligned}
$$

\subsection{Choice of reparametrization}

In order to eliminate the contribution containing $\left(w^{\prime}\right)^{2}$ we have to fix $g(w)$ such that $g(w)=$ $C f^{(1)}(w), C \in \mathbb{C}$. The simplest choice is to take $C=1$ and

$$
g(w)=f^{(1)}(w)
$$

Then Eq. (39) becomes simply:

$$
w^{\prime \prime}+\frac{2}{m} f^{(1)}(w) \frac{\partial}{\partial \bar{w}}\left(\overline{f^{(1)}(w)}(\widetilde{U}(w, \bar{w})-E)\right)=0 .
$$

Defining:

$$
V_{f}(w, \bar{w})=\left|f^{(1)}(w)\right|^{2}(\widetilde{U}(w, \bar{w})-E)+V_{0},
$$

where $V_{0}$ is an arbitrary real constant, we finally obtain

$$
w^{\prime \prime}+\frac{2}{m} \frac{\partial V_{f}(w, \bar{w})}{\partial \bar{w}}=0 .
$$

In other words, $w(s)$ is the motion of a particle of mass $m$ in the potential $V_{f}(w, \bar{w})$. If we choose as supplementary additive term an arbitrary holomorphic function $V_{0}(w)$, we are led to the same equation, but the corresponding potential $V_{f}(w, \bar{w})$ is no more real valued.

As for the energy of this dual system, it is given by:

$$
\widetilde{E}=\frac{m}{2}\left|w^{\prime}\right|^{2}+V_{f}(w, \bar{w})=V_{0} .
$$

Relation (42) becomes finally:

$$
\left|f^{(1)}(w)\right|^{2}=\frac{\widetilde{E}-V_{f}(w, \bar{w})}{E-\widetilde{U}(w, \bar{w})}=\frac{\widetilde{E}-V_{f}(w, \bar{w})}{E-U(f(w), \bar{f}(\bar{w}))} .
$$


We then obtain a functional equation for the transformation $f$ linking the motions in the potentials $U$ and $V$, respectively.

In conclusion, among all the transformations of a 2D-motion in a potential $U$ combining a conformal (i.e. analytical) change of coordinates $z=f(w)$ and an Euler-Sundman reparametrization $d t=|g(w)|^{2} d s$, those for which the transformed motion is an autonomous 2D-motion submitted to a potential $V$, necessarily satisfy Eqs. (40) and (45).

\subsection{Arnold-Vassiliev's potentials}

We consider the case in which the initial potential $U(\vec{r})$ takes the form $U(\vec{r})=U(z, \bar{z})=$ $A \overline{u(z)} u(z)=A|u(z)|^{2}$, where $u(z)$ is an analytical function on $\mathbb{C}^{*}$ and $A \in \mathbb{R}$. We call such a potential an Arnold-Vassiliev potential [2]. It is easy to show that the only central ArnoldVassiliev potentials are the power law potentials, corresponding to $u(z) \sim z^{\nu / 2}, \nu \in \mathbb{R}$, that is:

$$
U(z, \bar{z})=A|z|^{\nu}, \quad \nu, A \in \mathbb{R} .
$$

If $\widetilde{u}(w)=(u \circ f)(w)$, we have, by the transformation associated to $f$, an associated dual potential of the form (see Eq. (45)):

$$
\widetilde{E}-V(w, \bar{w})=\left|f^{(1)}(w)\right|^{2}\left(E-A|\widetilde{u}(w)|^{2}\right) .
$$

The question is now to determine for which type of conformal transformation, $f$, the corresponding image potential $V$ is also an Arnold-Vassiliev potential $V(w, \bar{w})=B|v(w)|^{2}$. This is clearly verified for the choice:

$$
w=f^{-1}(z)=\int u(z) d z
$$

or

$$
v(w)=f^{(1)}(w)=\frac{1}{u(f(w))}=\frac{1}{u(z)}
$$

with

$$
\widetilde{E}=-A, \quad B=-E .
$$

The transformations satisfying Eqs. (48) and (50) conserve the set of Arnold-Vassiliev potentials. Since they are involution, they can be seen as duality transformations:

$$
\left(E, A|u(z)|^{2}\right)^{w=\int} \underset{u(z) d z}{\rightarrow}\left(-A, \frac{-E}{|u(z)|^{2}}\right) \stackrel{\zeta=\int v(w) d w=z}{\longrightarrow}\left(E, A|u(z)|^{2}\right) .
$$

The Arnold-Vassiliev potentials appear then as constituting the more natural class of potentials for which the above combination of an analytical change of coordinates and an Euler-Sundman reparametrization is a dual correspondence. 


\subsection{Power-law potentials}

Consider the particular case of power-law potentials: $u(z)=z^{\frac{\nu}{2}}, U(z, \bar{z})=A|z|^{\nu}$, at fixed energy E. Equation (8) becomes:

$$
\ddot{z}+\frac{\nu A}{m}|z|^{\nu-2} z=0 \text {. }
$$

The preceding results (see Eq. (51)) give:

$$
\left\{\begin{array}{l}
w=f^{-1}(z)=\frac{1}{1+\nu / 2} z^{\nu / 2+1} \\
z=f(w)=\left(1+\frac{\nu}{2}\right)^{1 /(1+\nu / 2)} w^{1 /(1+\nu / 2)} .
\end{array}\right.
$$

By the duality transformation considered above the corresponding image potential is then given by:

$$
v(w)=\left(1+\frac{\nu}{2}\right)^{-\nu /(\nu+2)} w^{-\nu /(\nu+2)}, \quad V(w, \bar{w})=B|v(w)|^{2}=\widetilde{A}|w|^{\mu},
$$

where:

$$
\mu=-\frac{\nu}{1+\nu / 2}, \quad \widetilde{A}=-E\left(1+\frac{\mu}{2}\right)^{-\mu} .
$$

The energy for the dual motion is $\widetilde{E}=-A$ and the equation of the dual image motion takes the form:

$$
w^{\prime \prime}+\frac{\mu \widetilde{A}}{m}|w|^{\mu-2} w=0 .
$$

We recover for the dual image motion, a motion of energy $(-A)$, submitted to a power law potential of characteristic exponent $\mu$, such that:

$$
\left(1+\frac{\nu}{2}\right)\left(1+\frac{\mu}{2}\right)=1
$$

and for which the coupling constant is proportional to the opposite of the initial energy $E$ (and real if $\nu>-2$ ).

The relation between the two motions will be called "Bohlin-Arnold-Vassiliev duality".

In the special case $\nu=2$, we obtain $\mu=-1$. In other words, the dual motion of the planar harmonic oscillator (Hooke potential $\frac{1}{2} k r^{2}$, energy $E>0$ ) is nothing but the Kepler motion (Newton potential $-E / 2 \rho$, energy $\widetilde{E}=-k / 2<0$ ). We recover the usual Levi-Civita regularizing transformation $[22]$ with the associated change of variables:

$$
z=\sqrt{2 w}, \quad w=\frac{1}{2} z^{2}
$$

and reparametrization:

$$
d s=2 \rho d t=r^{2} d t
$$

where $r=|z|$ and $\rho=|w|$. 


\subsection{Bohlin-Arnold-Vassiliev's duality and conserved quantities}

For a general 2D-central potential we have two real conserved quantities: the energy $E$, associated to translational invariance in time, and the angular momentum $L$, associated to rotational invariance. In the case of power-law potentials the effect of the duality transformation on the energy is given by Eq. (55). Concerning the angular momentum it is easy to show that (see Eq. (40)):

$$
L=\frac{m}{2 i}(\bar{z} \dot{z}-\dot{\bar{z}} z)=\frac{m}{2 i}\left(\frac{w^{\prime}}{\overline{(\log f(w))^{(1)}}}-\frac{\bar{w}^{\prime}}{(\log f(w))^{(1)}}\right)
$$

which gives for power law potentials (see Eq. (53)):

$$
L=\left(1+\frac{\mu}{2}\right) \widetilde{L}
$$

where $\widetilde{L}$ is the angular momentum of the $w(s)$ motion.

Among all the central potentials, only the Kepler system and the isotropic harmonic oscillator possess true additional conserved quantities which are the Laplace-Runge-Lenz vector $\mathcal{A}[21]$ and the Jauch-Hill-Fradkin tensor $\mathcal{T}$ (or $\mathcal{F}$ ) [7, 19] respectively.

With all the other central potentials are associated only piecewise conserved vectors $[12,18]$.

In terms of the $w$ variable and $s$ parameter (see Eqs. (58) and (59)) $\mathcal{F}$ takes the form:

$$
\mathcal{F}=\frac{m}{4 w}(\dot{w})^{2}+k w=m \bar{w}\left(w^{\prime}\right)^{2}+k w .
$$

Since the energy of the dual motion is given by $\widetilde{E}=-k / 2=\frac{1}{2} m\left|w^{\prime}\right|^{2}-\widetilde{k} / \rho$, where $\widetilde{k}=E / 2>0$, we can write

$$
\mathcal{F}=m w^{\prime}\left(\bar{w} w^{\prime}-w \bar{w}^{\prime}\right)+E \frac{w}{\rho}=2 i w^{\prime} \widetilde{L}+2 \widetilde{k} \frac{w}{\rho},
$$

that is

$$
\mathcal{F}=2 \mathcal{A},
$$

where

$$
\mathcal{A}=\frac{m}{\widetilde{k}} i w^{\prime} \widetilde{L}-\frac{w}{\rho}
$$

is the affix of the well-known Laplace-Runge-Lenz vector [21] for the Kepler motion $\vec{\rho}(s) \rightarrow$ $w(s)$ :

$$
\vec{A}=\overrightarrow{\widetilde{L}} \times \vec{\rho}^{\prime}+\widetilde{k} \frac{\vec{\rho}}{\rho} .
$$

Consequently, the LRL vector and the JHF tensor, or more precisely the generators of the dynamical symmetry algebras of the Kepler system and the isotropic harmonic oscillator respectively, are (up to a constant factor) two forms of the same object, expressed in the systems of representation (coordinates and parametrization) corresponding to each of the dual motions. 
It is interesting to note that the elementary approach developed here can be extended to a much more general set of planar motions conserving only the angular momentum direction $[9,11]$. In this case, the duality connects different classes of motions. For those presenting closed orbits which satisfy generalized Gorringe-Leach equations, there exist additional conserved quantities which are linked by a relation similar to Eq. (62).

\section{Conclusion}

The interest of the use of complex variable in the study of planar systems has already been largely emphasized $[1,6,25,32]$. Our main aim in this paper was to highlight a more unusual aspect, namely its specific relevance concerning the interpretation of dynamical conserved quantities. We have shown that in a complex variable formulation, the existence of such a conserved quantity, namely the JHF tensor, is almost trivially induced by the particular structure of the equation of motion. Using the Bohlin-Arnold-Vassiliev duality we have then shown that the LRL vector and the JHF tensor are obtained from the same vector, expressed in the dual dynamical representation systems of the Kepler and isotropic harmonic oscillator respectively.

\section{References}

[1] V. I. Arnold, Huygens and Barrow, Newton and Hooke (Birkhäuser, Basel, 1990).

[2] V. I. Arnold and V. A. Vassiliev, Newton's principia read 300 years later, Not. Am. Math. Soc. 36 (1989) 1148-1154; 37 (1990) 144 (addendum).

[3] J. Bertrand, Théorème relatif au mouvement d'un point attiré vers un centre fixe, C. R. Acad. Sci. 77 (1873) 849-853.

[4] M. K. Bohlin, Note sur le problème des deux corps et sur une intégration nouvelle dans le problème des trois corps, Bull. Astron. 28 (1911) 113-119.

[5] P. Collas, Equivalent potentials in classical mechanics, J. Math. Phys. 22 (1981) 2512-2517.

[6] R. W. Finkel, Complex coordinates in central force analyses, Am. J. Phys. 58 (1990) 1085.

[7] D. M. Fradkin, Three-dimensional isotropic harmonic oscillator and SU3, Am. J. Phys. 33 (1965) 207-211.

[8] H. Goldstein, More on the prehistory of the Laplace or Runge-Lenz vector, Am. J. Phys. 44 (1976) 1078-1082.

[9] V. M. Gorringe and P. G. L. Leach, Kepler's third law and the oscillator's isochronism, Am. J. Phys. 61 (1993) 991-995.

[10] Y. Grandati, A. Bérard and F. Menas, An alternative proof of Bertrand's theorem, Am. J. Phys. 76 (2008) 782-787.

[11] Y. Grandati, A. Bérard and H. Mohrbach, Duality properties of generalized Gorringe-Leach equations, Celest. Mech. 103 (2009) 133-141.

[12] Y. Grandati, A. Bérard and H. Mohrbach, On Peres approach to Fradkin-Bacry-RueggSouriau's perihelion vector [arXiv:0810.3780].

[13] A. K. Grant and J. L. Rosner, Classical orbits in power-law potentials, Am. J. Phys. 62 (1994) 310-315.

[14] D. F. Greenberg, Accidental degeneracy, Am. J. Phys. 34 (1966) 1101-1109.

[15] R. W. Hall and K. Josić, Planetary motion and the duality of force laws, SIAM. Rev. 42 (2000) $115-124$.

[16] J. Hietarinta, B. Grammaticos, B. Dorizzi and A. Ramani, Coupling-constant metamorphosis and duality between integrable Hamiltonian systems, Phys. Rev. Lett. 53 (1984) 1707-1710.

[17] S. A. Hojman, S. Chayet, D. Nunez and M. A. Roque, An algorithm to relate general solutions of different bidimensional problems, J. Math. Phys. 32 (1991) 1491-1497. 
[18] A. Holas and N. H. March, A generalisation of the Runge-Lenz constant of classical motion in central potential, J. Phys. A 23 (1990) 735-749.

[19] J. M. Jauch and E. L. Hill, On the problem of degeneracy in quantum mechanics, Phys. Rev. 57 (1940) 641-645.

[20] E. Kasner, Differential-geometric aspects of dynamics, in The Princeton Colloquium (AMS, Providence, 1913).

[21] P. G. L. Leach and G. P. Flessas, Generalisations of the Laplace-Runge-Lenz vector, J. Nonlin. Math. Phys. 10 (2003) 340-423, and references therein.

[22] T. Levi-Civita, Sur la résolution qualitative du problème des trois corps, Acta. Math. 30 (1906) $305-327$.

[23] H. V. McIntosh, Symmetry and degeneracy, in Group Theory and Its Applications, ed. E. M. Loebl (Academic, New York, 1971), Vol II, p. 82.

[24] T. P. Mitchell, Conformally dual fields in relativistic classical and quantum mechanics, Am. J. Phys. 64 (1996) 1158-1160.

[25] L. Mittag and M. J. Stephen, Conformal transformations and the application of complex variables in mechanics and quantum mechanics, Am. J. Phys. 60 (1992) 207-211.

[26] T. Needham, Visual Complex Analysis (Oxford University Press, New York, 1997).

[27] T. Needham, Newton and the transmutation of forces, Am. Math. Monthly 100 (1993) 119-137.

[28] A. Nersessian and G. Pogosyan, Relation of the oscillator and Coulomb systems on spheres and pseudospheres, Phys. Rev. A 63 (2001) 020103R.

[29] A. Nersessian and V. Ter-Antonyan, Anyons, monopole and Coulomb problem, 8th Conference on Symmetry Methods in Physics (Dedicated to 80th Birthday of Pr. Smorodinsky), Dubna, Russia, 28 Jul-2 Aug 1997, Phys. Atom. Nucl. 61 (1998) 1756.

[30] A. Nersessian, V. Ter-Antonyan and M. Tsulaia, A note on quantum Bohlin transformation, Mod. Phys. Lett. A 11 (1996) 1605.

[31] K. Rosquist and G. Pucacco, Invariants at fixed and arbitrary energy. A unified geometric approach, J. Phys. A 28 (1995) 3235-3252.

[32] D. G. Saari, A visit to the Newtonian N-body problem via elementary complex variables, Am. Math. Monthly 97 (1990) 105-119.

[33] J. Sivardière, Comments on the dynamical invariants of the Kepler and harmonic motions, Eur. J. Phys. 13 (1992) 64-69.

[34] E. Stiefel and G. Scheifele, Linear and Regular Celestial Mechanics (Springer, Berlin, 1971).

[35] D. R. Stump, Arnold's transformation - An example of a generalized canonical transformation, J. Math. Phys. 39 (1998) 3661-3669.

[36] D. R. Stump, A solvable non-central perturbation of the Kepler problem, Eur. J. Phys. 19 (1998) 299-306.

[37] A. V. Tsiganov, The Kepler canonical transformations of the extended phase space, Reg. Chao. Dyn. 5 (2000) 117-127.

[38] H. Wu and D. W. L. Sprung, Comment on "Classical orbits in power-law potentials", Am. J. Phys. 63 (1995) 564-567. 